\title{
ABOUT DUAL SPHERICAL WRIST MOTION AND ITS TRAJECTORY SURFACE AS A RULED SURFACE
}

\author{
Hatice Kuşak $^{1}$, Ali Çalışkan ${ }^{2}$ \\ Department of Mathematic, Ege University, 35030, Bornova, İzmir, Turkey \\ 1ecitah_tamus@yahoo.com, ${ }^{2}$ ali.caliskan@ege.edu.tr
}

\begin{abstract}
Solid object rotations or rotations of unit real vectors are often used in robotic moves. Yet, due to the complexity of calculations in kinematics analysis, quaternions began to be used in robotic moves. Quaternions help us to calculate rotations in reel space, and rotation and translation in dual space. Furthermore, we can easily define a screw or an axis through dual vectors. In recent years, there has been efficient studies in mechanics analysis by using dual quaternions and screw moves. In this study, we transferred the well known sequential and modular methods for the rotations being formed in real spherical wrist moves to dual space. Hence, while we rotate the unit vectors only in real space, now we can rotate screws .Hence, while we rotate the unit vectors only in real space, now we can rotate screws or the axis in the dual spherical mechanisms in dual kinematics. Through this study, by being switched to dual spherical mechanism the first time, we will see that we can carry out what we do with unit vectors in real space as well as in dual space. Furthermore, while the orbits drawn by the joint moves are curves in reel spherical wrist moves, the orbits drawn by the joint moves are ruled surfaces in dual spherical wrist moves. We think that this situation would establish a base for different mechanics studies.
\end{abstract}

Key Words- Dual Wrist Motion, Ruled Surface, Screw

\section{INTRODUCTION}

Quaternions are commonly used in science fields such as physics, chemistry, robotic, mechanical, electronic because of their practicality.

Hypercomplex numbers allow simplifying the practical calculations in a drastic way. At the same time they are applied to such problems of modern computational kinematics, [6]. $q=a+b \vec{i}+c \vec{j}+d \vec{k}$ quaternion can rotate the real vector or points of a rigid body in matris form. Rotation matris has a complex structure but this difficulty removed with the quaternion's simple structure. Sir W.R Hamilton was led by geometrical applications: if motion in one direction along a line be treated as positive motion in the opposite direction along the same line is negative. The topological geometry in spatial kinematics had been discussed in [3], and the representation of spherical displacements and motions are described by rotation group of unit quaternions. P.E Nixravesh [4] introduces the method which is based on a sequence of matrix computation, and identities for relating a representation of spherical motion with their corresponding velocity and acceleration vectors. R. Larochelle [5], uses planar quaternions to create syntheurs equations for planar robots, and created a virtual reality environment that could promote the design of spherical manipulators. In paper [7] authors described the physical model of the solution space for the spherical 3-DOF serial wrists. Also, writers 
are improved a new method in [6] reference for kinematical calculations of the spherical wrist motion by using quaternions. In this study, quaternion operator which requires the rotation of rigid bodies or unit real vectors, are inadequate for applying of rotation and translation. In such situations, the $\mathrm{A}, \mathrm{B}, \mathrm{C}, \mathrm{D}$ dual numbers are preferred by using $\mathrm{Q}=\mathrm{D}+\mathrm{A} \overrightarrow{\mathrm{i}}+\mathrm{B} \overrightarrow{\mathrm{j}}+\mathrm{C} \overrightarrow{\mathrm{k}}$ dual quaternions. Especially, rotation and translation of dual vectors or axodes are more practical and useful in space kinematic and robotic. Veldkamp and Yang- Freudenstein investigated the use of dual numbers matrix and dual quaternions in instantaneous spatial kinematics in [9] and [8], respectively. In [8], an application of dual quaternion algebra to the analysis of spatial mechanism was given. Agrawal [1] worked on Hamilton operators and dual quaternions in kinematics.

In the paper [2] the representation is based on the screw displacement pair, i.e. , the dual number extension of the rotational displacement pair and consists of the dual unit vector of the screw axis and the associate dual angle of the amplitude.We try to survey spherical wrist motion which is well known in robotic and mechanical studies on dual space. While rotating the quaternions and unit vectors, we will use dual quaternion for rotating lines and screws. So, it will form the dual spherical wrist motion. Another advantage of studying dual space is being a curve that occured by a mechanism with real spherical wrist motion, on the other hand, it is an orbit that occured by a mechanism with dual spherical wrist motion.

It is know that ruled surface have very important roles on the area of usage in space kinematic. In our study we composed of advantages of computer with robotic, mechanic, theorical geometry and CAGD, also composed of a common practice space which is used for those areas.

\section{DUAL QUATERNIONS AS A PRODUCT OF TWO VECTOR}

We can define $+1, \overrightarrow{\mathrm{i}}, \overrightarrow{\mathrm{j}}, \overrightarrow{\mathrm{k}}, \varepsilon, \varepsilon \overrightarrow{\mathrm{i}}, \varepsilon \overrightarrow{\mathrm{j}}, \varepsilon \overrightarrow{\mathrm{k}}$ elements as a basic elements from the $\mathrm{Q}=\mathrm{d}+\mathrm{a} \overrightarrow{\mathrm{i}}+\mathrm{b} \overrightarrow{\mathrm{j}}+\mathrm{c} \overrightarrow{\mathrm{k}}+\mathrm{d}^{*} \varepsilon+a^{*} \varepsilon \overrightarrow{\mathrm{i}}+\mathrm{b}^{*} \varepsilon \overrightarrow{\mathrm{j}}+\mathrm{c}^{*} \varepsilon \overrightarrow{\mathrm{k}}$

dual quaternion equation. In here $d, a, b, c, a^{*}, b^{*}, c^{*} \in I R$ and

$$
\overrightarrow{\mathrm{i}}^{2}=\overrightarrow{\mathrm{j}}^{2}=\overrightarrow{\mathrm{k}}^{2}=\overrightarrow{\mathrm{ij}} \overrightarrow{\mathrm{k}}=-1, \quad \varepsilon^{2}=0
$$

conditions are provided. Here for following dual numbers

$$
\mathrm{D}=\mathrm{d}+\varepsilon \mathrm{d}^{*}, \quad \mathrm{~A}=\mathrm{a}+\varepsilon a^{*} \quad \mathrm{~B}=\mathrm{b}+\varepsilon b^{*} \quad \mathrm{C}=\mathrm{c}+\varepsilon c^{*}
$$

a dual quaternion can be written as the combination of dual vectors;

$$
\mathrm{Q}=\mathrm{D}+\mathrm{A} \overrightarrow{\mathrm{i}}+\mathrm{B} \overrightarrow{\mathrm{j}}+\mathrm{C} \overrightarrow{\mathrm{k}}
$$

Also, we can define a dual quaternion as a dual quaternion multiplication of two dual vectors. So, we can define

$$
Q=\left(A_{1} \vec{i}+B_{1} \vec{j}+C_{1} \vec{k}\right) \cdot\left(A_{2} \vec{i}+B_{2} \vec{j}+C_{2} \vec{k}\right)
$$

as $Q$ dual quaternion. $\vec{R}_{1}, \vec{R}_{2}$ and $\vec{R}$ be the unit distances. $\theta$ is rotation angle between $\vec{R}_{1}$ and $\vec{R}_{2}$ unit vectors and $\theta^{*}$ is the translation angle. So, we define $\Theta=\theta+\varepsilon \theta^{*}$ as the dual angle, in Fig 1. 


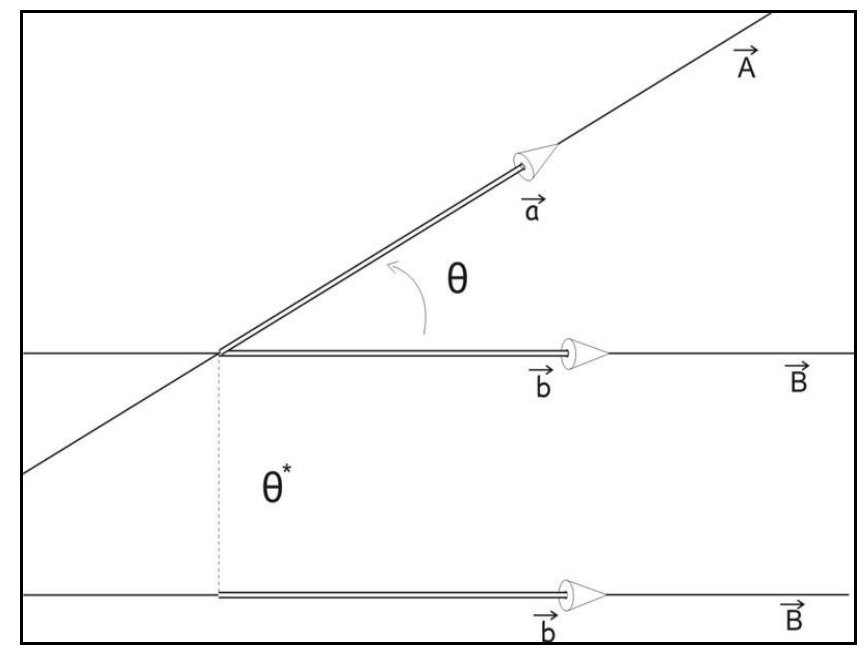

Figure 1- Dual angle

If $\vec{R}_{3}$ vector is perpendicular to $\vec{R}_{1}$ and $\vec{R}_{2}$ vectors then the dual quaternion multiplication of $\vec{R}_{1}$ and $\vec{R}_{2}$ dual vectors composed of a unit dual quaternion in (6).

$$
\begin{aligned}
\mathrm{Q} & =\overrightarrow{\mathrm{R}}_{1} \times \overrightarrow{\mathrm{R}}_{2}=\left(\mathrm{A}_{1} \overrightarrow{\mathrm{i}}+\mathrm{B}_{1} \overrightarrow{\mathrm{j}}+\mathrm{C}_{1} \overrightarrow{\mathrm{k}}\right) \cdot\left(\mathrm{A}_{2} \overrightarrow{\mathrm{i}}+\mathrm{B}_{2} \overrightarrow{\mathrm{j}}+\mathrm{C}_{2} \overrightarrow{\mathrm{k}}\right) \\
& =-<\overrightarrow{\mathrm{R}}_{1}, \overrightarrow{\mathrm{R}}_{2}>+\overrightarrow{\mathrm{R}}_{1} \wedge \overrightarrow{\mathrm{R}}_{2} \\
& =-\left|\overrightarrow{\mathrm{R}}_{1}\right| \cdot\left|\overrightarrow{\mathrm{R}}_{2}\right| \cdot \cos \Theta+\left|\overrightarrow{\mathrm{R}}_{1}\right| \cdot\left|\overrightarrow{\mathrm{R}}_{2}\right| \cdot \sin \Theta \cdot \vec{M} \\
& =-\cos \Theta+\vec{M} \cdot \sin \Theta
\end{aligned}
$$

In here, $\vec{R}_{3}$ vector is overlapped with $\vec{M}=\vec{m}+\approx \vec{m}^{*}$ unit screw straight linet hat means $\vec{R}_{n}=\vec{M}$. So, it is possible to show an unit dual quaternion as in (7)

$$
\mathrm{Q}=-\cos \Theta+\vec{M} \cdot \sin \Theta
$$

\section{ROTATING A SCREW VIA DUAL QUATERNION}

The unit real quaternion $q=a+b \vec{k}+c \vec{j}+d \vec{k}$ makes a vector rotate around an axis with an angle in real space. It is seen that quaternions are widely used in engineering, mechanics, and especially in robotics studies. However, if the structure we want to transform was not a vector but a screw or an axis, then the real quaternions would not be sufficient in transforming these structures. In robotic moves, both the rotation and the translation moves of screws or axis are simultaneously done in a transformation in unit dual quaternions. Unit dual quaternion

$$
\mathrm{Q}=-\cos \Theta+\vec{M} \cdot \sin \Theta
$$

is given with this equation in (8). Let $\Theta=\theta+\varepsilon \theta^{*}$ be the dual angle and $\vec{M}=\vec{m}+\approx \vec{m}^{*}$ be the screw straight line. And $\mathrm{Q}^{-1}=-\cos \Theta-\vec{M} \cdot \sin \Theta$ is the opposite of $q$ dual quaternion. Also we can show the rotation multiplication of $\vec{R}=\vec{r}+\varepsilon \vec{r}$ screw with $Q$ dual quaternion as $Q(\vec{R}) Q^{-1}$. With this impression $\vec{R}$ screw moves two times with $\Theta$ angle. 


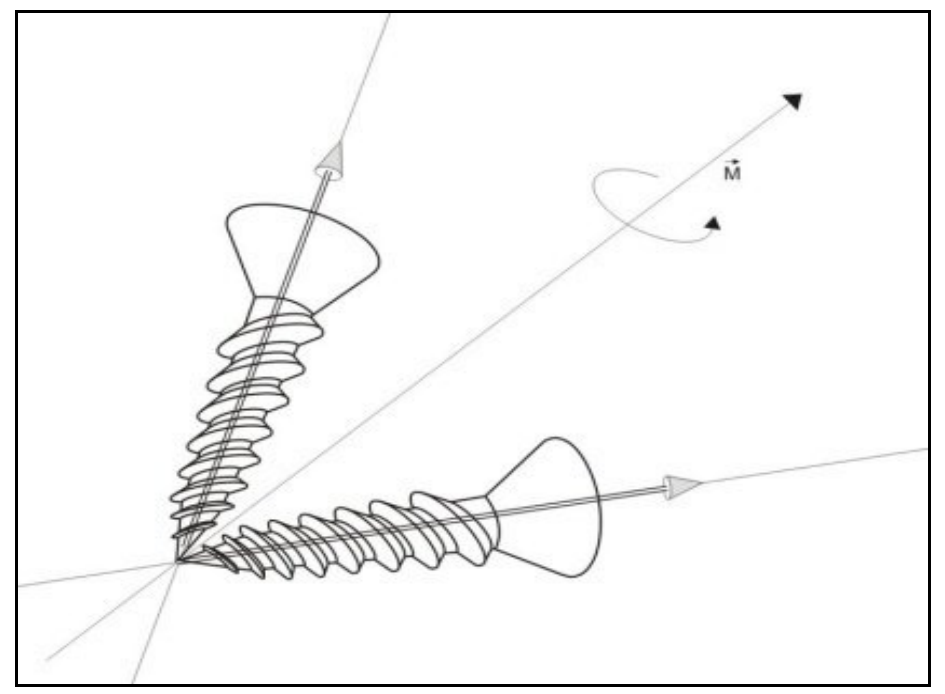

Figure 2 -Rotation of a screw

By using this multiplication, for making one screw motion $q$ dual quaternion will be like that in (9);

$$
\mathrm{Q}=-\cos \frac{\Theta}{2}+\vec{M} \cdot \sin \frac{\Theta}{2} \text {. }
$$

The dual angle $\oplus$ both rotates and translates the screws. Yet, since our study is about

dual sphere wrist moves, we will take the dual angle as $\Theta^{*}=0$ and use the dual quaternion which contains only a rotation operator. Therefore, we obtain $\Theta=0$ and apply a rotation to a screw or an axis through the quaternion $\mathrm{Q}=\cos \frac{\Theta}{2}+\vec{M} \cdot \sin \frac{\Theta}{2}$, see in Fig 2. At the same time, let us make $\vec{R}$ an axis by taking it as unit.

\section{SEQUENTIAL METHOD WITH MOVEMENTS OF SCREW ROTATION DETERMINED BY A DUAL QUATERNION}

In order to rotate screws or axis with sequential method via dual quaternion, the axis $\vec{R}=\vec{r}+s \vec{r}^{*}$ should rotate around the axis $\vec{M}_{i}$ with the dual angle $\Theta_{i}$. Then, in sequential method, every screw $\vec{R}_{i}, i=1,2, \ldots, n$, can be transformed to the screw $\vec{R}_{i+1}$, in Fig 3.In other words, the sequential method can be defined as in (10)

$$
\vec{R}_{i+1}=Q_{i}\left(\vec{R}_{i}\right) Q_{i}^{-1} \text {. }
$$

Generalized as $\vec{R}_{1}$ screw being sequential $Q$ dual quaternion application is same with the given equation

$$
\vec{R}_{n+1}=Q_{n} Q_{n-1} \ldots Q_{2} Q_{1}\left(\vec{R}_{i}\right) Q_{1}^{-1} Q_{2}^{-1} \ldots Q_{n-1}^{-1} Q_{n}^{-1} .
$$




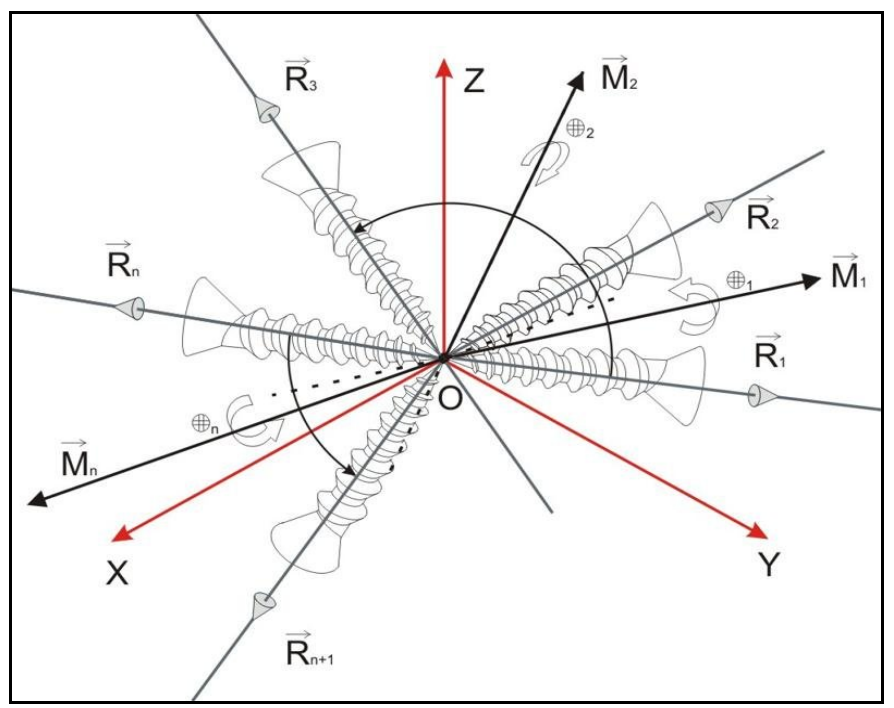

Figure 3-Sequential method via dual quaternion

\section{THE MODULAR METHOD DEFINED WITH A ROTATION OF A DUAL QUATERNION}

The dual quaternions that produce the screw motions are widely used in robotic motions. For example, let us consider the factory in which the assembling of the automobile parts are done. In order to implement rotation and translation to every single piece, the transformation should be applied to the parts in a certain order. In order to do this, according to our aim of applying the dual quaternion, we can use modular method along with the sequential method.

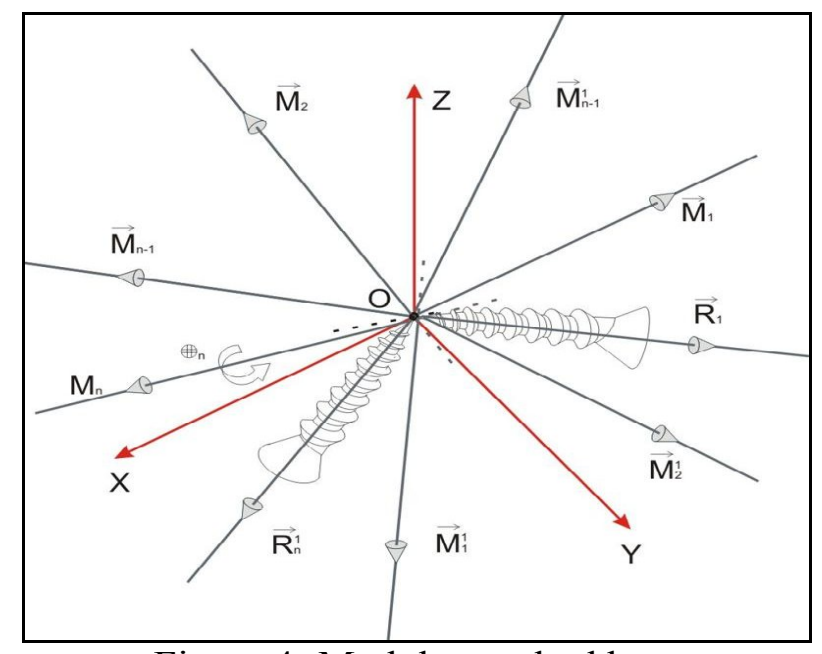

Figure 4 -Modular method by screws

In our study, since we will deal with the dual spherical wrist move, we take the dual quaternion as rotation operator. We define the modular method in dual spherical wrist move as following: 
Let the screw $\vec{R}_{1}$ and the axis $\vec{M}_{1}$ are rotated around the axis $\vec{M}_{\mathrm{n}}$ with the angle $\Theta_{n}$, as in Fig.4. Let us represent the rotated screw with $\vec{R}_{1}^{1}$ and the new axis with $M_{1}^{1}$. Let us rotate the newly formed screw and axis around the axis $\vec{M}_{n 2}$ again with the angle $\Theta_{n}$. Let us continue to repeat this process until we obtain the screw $\overrightarrow{\mathbb{R}}_{1}^{n}$. In other words, let us define the dual quaternion as in (11)

$$
\mathrm{Q}_{\mathrm{i}}^{*}=-\cos \frac{\Theta_{k}}{2}+\hat{m}_{k}^{i-1} \cdot \sin \frac{\Theta_{k}}{2},
$$

Here $k=p+1-i$ and $\mathrm{i}=1,2, \ldots, \mathrm{n}$. If we rotate the screw $\overrightarrow{\boldsymbol{R}}_{1}$ via the dual quaternion $q_{i}$ with the angle $\Theta_{k}$, we obtain the screws in (12)

$$
\vec{R}_{1}^{i}=Q_{i}^{*}\left(\vec{R}_{1}\right) Q_{i}^{*-1}
$$

Likewise, if we rotate the axis $\vec{M}_{\bar{i}}$ via the dual quaternion $\ell_{i}$ with the angle $\Theta_{k}$ we obtain the axis as (13)

$$
\vec{M}_{j}^{i}=Q_{i}^{*}\left(M_{j}^{i-1}\right) Q_{i}^{*-1}
$$

Therefore, we produce the modular method for dual spherical wrist moves which are defined with the rotation of dual quaternion as following:

$$
\vec{R}_{1}^{n}=Q_{n}^{*} Q_{n-1}^{*} \ldots Q_{2}^{*} Q_{1}^{*}\left(\vec{R}_{i}\right) Q_{1}^{*-1} Q_{2}^{*-1} \ldots Q_{n-1}^{*-1} Q_{n}^{*-1} .
$$

\section{WRIST MOTIONS DEFINED BY DUAL QUATERNIONS IN DUAL SPHERICAL MECHANISM}

As it is known, the spherical wrist moves in spherical mechanisms are widely used in mechanical engineering. The rotation of unit vectors with real angles in the spherical moves of real space is possible. In the spherical mechanism, the axis which is drawn by the spherical wrist move on real sphere consists of spherical curves, see in Fig.5. This results in various applications in mechanics.

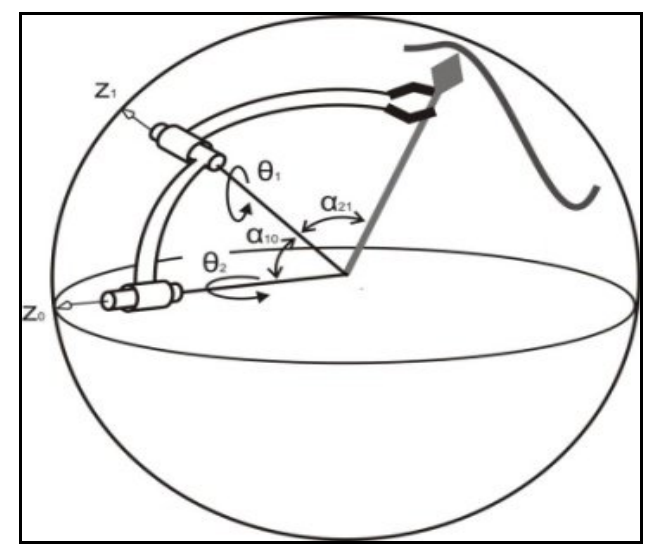

Figure5- Reel spherical wrist motion and its trajectory as a curve

In our study, since we use the transformation of lines, we cannot work with the quaternions in real space. Therefore, since a unit dual vector indicates a line (axis) in dual space, we need to deal with the move of a screw via dual quaternion. The result of transforming dual vectors through sequential or modular methods is a dual sphere. 
Therefore, the wrist move defined with dual quaternions is described in spherical mechanisms. As it is shown in the Fig 5, let us assume that the 2DOF dual spherical manipulator is given. Here, we will analyze the last position of the robot arm through the sequential or modular method.

First, let us analyze the position of the robot arm after the first rotation via the use of sequential method. We will determine the last position of $\vec{R}_{2}$ screw that rotates with $\Theta_{1}$ dual angle around $\vec{R}_{1}$, later we will rotate $\vec{R}_{2}$ screw with $\Theta_{2}$ angle around $\vec{R}_{0}$ screw as in (14).

$$
\vec{R}_{2}^{1}=Q_{1}\left(\vec{R}_{1}\right) Q_{1}^{-1} .
$$

In here, it is formed like $\mathrm{Q}_{1}=-\cos \frac{\Theta_{1}}{2}+\vec{R}_{1} \cdot \sin \frac{\Theta_{1}}{2}$, later

$$
\vec{R}_{2}^{2}=Q_{2}\left(\vec{R}_{2}^{1}\right) Q_{2}^{-1} .
$$

occurs in (15), and here $\mathrm{Q}_{2}=-\cos \frac{\Theta_{2}}{2}+\vec{R}_{0} \cdot \sin \frac{\Theta_{2}}{2}$. If we combine these two equations, the last position of the robot arm which is formed by the sequential method is in (16)

$$
\vec{R}_{2}^{2}=Q_{2} Q_{1}\left(\vec{R}_{2}\right) Q_{1}^{-1} Q_{2}^{-1} .
$$

\section{ON DUAL SPHERICAL WRIST MOTIONS TRAJECTORY SURFACE AS RULED SURFACE}

There is a spherical curve on real sphere which is rotating each robotic arm or joint during motion is drawn by the real spherical wrist motion by the help of quaternion rotation. We know that those orbit curves are commonly used in kinematic analysis in engineering mechanisms. When dual kinematic passes to the space, the elements of kinematic has different meanings.

In our study we can easily see that the robot's arms or joints are drawing dual curves on the dual sphere. A point can correspond to a line on dual sphere and also a curve can correspond to a ruled surface. So that, in dual spherical motions, every dual trajectory of the rotated straight lines or screw is called as ruled surface on dual sphere, in Fig.6. For dual quaternion $\mathrm{Q}=-\cos \frac{\Theta}{2}+\vec{M} \cdot \sin \frac{\Theta}{2}$, applied $\vec{R}$ screw is composed of the screw as $\vec{R}^{1}=Q(\vec{R}) Q^{-1}$. If we think of every $\mathrm{t}$ time for dual spherical wrist motion then

$$
\vec{R}^{1}(t)=Q(\vec{R}(t)) Q^{-1} .
$$

equation (17) gives us a ruled surface equation. So, we gain different view for ruled surface in kinematic. If the modular method is used, then for finding the last position of joint, when we rotate the $\vec{R}_{1}$ and $\vec{R}_{2}$ unit dual screws rotates with $\Theta_{2}$ angle around $\vec{R}_{\theta}$ with $\mathrm{Q}_{1}=-\cos \frac{\Theta_{2}}{2}+\vec{R}_{0} \cdot \sin \frac{\Theta_{2}}{2}$ dual quaternion, we get the unit dual screw. For being 2DOF, when we rotate the $\vec{R}_{2}^{1}$ screw with $\Theta_{1}$ dual angle that defined $\mathrm{Q}_{2}=-\cos \frac{\Theta_{1}}{2}+\vec{R}_{1}^{1^{*}} \cdot \sin \frac{\Theta_{1}}{2}$ dual quaternion, we get the lines at last position in (18):

$$
\vec{R}_{2}^{2^{*}}(t)=Q_{2}^{*}\left(\vec{R}_{2}^{1^{*}}\right) Q_{2}^{*-1}
$$


As seen from here, for defining the last position of robot's arm, we can establish the position by using sequential or modular methods with different procedures. Similar study can be applied on spherical mechanisms of 3DOF. And same results are found with generalized situations.

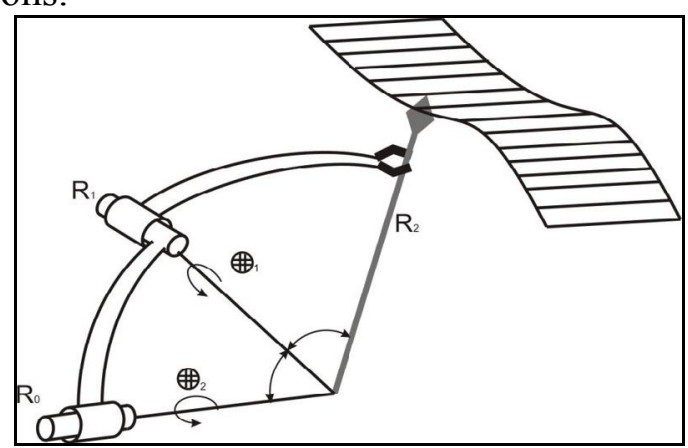

Figure 6- Dual spherical wrist motion with its trajectory as a ruled surfaces

\section{CONCLUDING REMARKS}

From this study we can understand that for anchoring robot arms, sequential and modular methods are used in dual kinematical space studies. Ruled surface has very important role in CAGD and robotic. Spherical wrist motions are used in many industrial machines and they produce spherical curves. In our study we are producing dual spherical wrist motions and ruled surface. So, our study can help the studies which has geometrical base that performs orbits in mechanical, robotic and CAGD of dual spherical wrist motion.

\section{REFERENCES}

1. Agrawal O.P., Hamilton Operators and Dual number-quaternions in Spatial Kinematics, Mech. Mach. Theory 22,569-575.

2. Hiller M., Woernle C., A unified representation of spatial displacements, Mech. Mach. Theory 19, 477-486, 1984.

3. I.R. Porteous, Topological Geometry, Cambridge University Press, U.K., 1921.

4. P.E. Nixravesh, R.A. Wehage and O.K. Kwan, Euler Parameters in Computational Kinematics and Dynamics, Part, ASME J. Mech. Trans, Aut. Des (107) 358-365, 1985.

5. P.Larochelle, Approximate Motion Synthesis via Parametric Constraint Manifold Fitting, Advances in Robot Kinematics, Kluwer Acad. Publ., Dordrecht, 2000.

6. Rasim I. Alizade, Erkin Gezgin, Özgür Kilit, A New Method in Computational Kinematics of a Spherical Wrist Motion Through Quaternions.

7. Xin-Jun Liu, Jinsong Wang and Fang Gao., Workspace Atlases fort he Design of Spherical 3-DOF Serial Wrist, J. Of Intelligent and Robotic Systems 389-405, 2003.

8. Yang A.T., Freudenstein F., Application of dual number quaternion algebra to the analysis of spatrial mechanism, Transactions of the ASNE , 300-308, 1964.

9. Veldkamp G.R, On the use of dual numbers, vectors and matrices in instantaneous, Spectral Kinametrics, Mech. Mach Theory 11,141-156, 1976. 